\title{
Effect of Vildagliptin Versus Sulfonylurea in Muslim Patients with Type 2 Diabetes Fasting During Ramadan in Egypt: Results from VIRTUE Study
}

\author{
Mohamed Khattab $\cdot$ Khalifa Mahmoud · Inass Shaltout
}

Received: May 5, 2016/ Published online: August 22, 2016

(C) The Author(s) 2016. This article is published with open access at Springerlink.com

\begin{abstract}
Introduction: Fasting in patients with type 2 diabetes mellitus (T2DM) is associated with high risk of hypoglycemia. The aim of this study was to compare the effectiveness and safety of vildagliptin in T2DM patients fasting during Ramadan in a real-life setting in Egypt.

Methods: In this 16-week prospective and noninterventional study, data were collected up to 6 weeks before and after Ramadan fasting. Patients who had received vildagliptin or sulfonylurea (SU) either as dual therapy with metformin or as monotherapy were enrolled into the study.
\end{abstract}

Results: Two hundred fifty four patients were enrolled in the study, out of which 246 [121

Enhanced content To view enhanced content for this article go to http://www.medengine.com/Redeem/ AAE4F06034380722.

M. Khattab $(\varangle) \cdot$ I. Shaltout Internal Medicine and Diabetes, Cairo University, Giza, Egypt

e-mail:m.khattab@hotmail.com

K. Mahmoud

Internal Medicine and Diabetes, Alexandria

University, Alexandria, Egypt
(97.6\%) treated with vildagliptin and 125 $(99.2 \%)$ with SU] were included in the safety analysis set. A significantly lower proportion of patients experienced $\geq 1$ hypoglycemic event (HE) with vildagliptin as compared to those receiving SUs $(1.7 \%$ vs. $19.2 \%$, respectively; $p<0.001)$. No patient in either group reported a grade $2 \mathrm{HE}$. At week 16, mean change in HbA1c from baseline for vildagliptin and SU were $-0.1 \%$ and $+0.3 \%$, respectively, with a between-treatment difference of $-0.4 \%$ $(p<0.001)$. Mean change in body weight from baseline for vildagliptin and SU were -0.8 and $-0.1 \mathrm{~kg}$, with a between-treatment difference of $-0.7 \mathrm{~kg} \quad(p=0.011)$. A higher proportion of SU-treated patients experienced adverse events compared to those treated with vildagliptin $(23.2 \%$ vs. $5.8 \%$, respectively), the primary reason being the high incidence of hypoglycemia in the SU group $(n=24,19.2 \%)$. Conclusions: Treatment with vildagliptin was associated with lower incidence of hypoglycemia compared with SU and showed good glycemic and weight control in patients with T2DM fasting during Ramadan in a real-life setting in Egypt.

Funding: Novartis Pharma AG. 
Keywords: Dipeptidyl peptidase-4 inhibitors; Hypoglycemia; Metformin; Ramadan; Type 2 diabetes; Vildagliptin

\section{INTRODUCTION}

The prevalence of diabetes mellitus has been increasing in Egypt. In 2014, over 7.5 million people (aged 20-79 years) were suffering from diabetes in Egypt and this number is expected to increase to 13.1 million by the year 2035 $[1,2]$. Approximately, over 80 million people in Egypt are Muslims and the majority of them choose to fast during Ramadan [3].

Ramadan is the holy month during which millions of Muslims across the world fast with complete faith and devotion. During this month, Muslims abstain from consuming food, beverages, and oral medications between sunrise and sunset for approximately 30 days $[4,5]$. Fasting is not meant to create excessive hardships for an individual; the Koran exempts some Muslims from fasting, which include pregnant or lactating women, the sick, and the elderly [6]. Consequently, both religious and medical advice suggests that people with diabetes should not fast since it could adversely affect their metabolic condition $[4,7]$. A large epidemiological study (EPIDIAR) conducted in 13 Muslim countries showed that fasting during Ramadan [8] reported an increased risk in hypoglycemia and severe hyperglycemia by 7.5-fold and fivefold, respectively, in patients with type 2 diabetes mellitus (T2DM) [4, 8, 9].

Several Muslims are unaware of the possible complications of fasting, which include hyperglycemia, hypoglycemia, and increased risk of dehydration and thrombosis [9, 10]. The American Diabetes Association developed a consensus statement on the management of diabetes during Ramadan, which recommended individualized management plan, close follow-up, and structured educational programs to reduce the risk of complications [4].

Vildagliptin, a potent and selective dipeptidyl peptidase-4 (DPP-4) inhibitor, improves glycemic control by increasing $\alpha$ and $\beta$-cell responsiveness to glucose $[11,12]$. Several long-term clinical trials have reported a decreased risk of hypoglycemia with vildagliptin [13]. Across different studies in T2DM patients who fast during Ramadan, vildagliptin has been shown to be effective, safe, well tolerated and associated with a low incidence of hypoglycemia, as well as good glycemic and weight control [14-20].

VildagliptIn expeRience compared wiTh sulfonylUreas obsErved during Ramadan (VIRTUE) was a large, prospective, observational, real-life study which pooled data from ten countries across the Middle East (Lebanon, Egypt, United Arab Emirates, Oman, Saudi Arabia, and Kuwait) and South Asia (Pakistan, India, Bangladesh, and Indonesia) [19]. This study reports the effectiveness and safety of vildagliptin as compared to sulfonylurea (SU) in Muslim patients with T2DM fasting during Ramadan. In view of limited data available, the present report would provide us an opportunity to look into the perspectives of real-life management of T2DM in fasting patients during Ramadan in Egypt.

\section{METHODS}

\section{Study Design and Patients}

This study was a multicenter, prospective, noninterventional, observational study 
conducted in Egypt which enrolled 254 patients at the beginning of Ramadan. Details of the study design have been previously presented elsewhere [19]. Patients included in the study were aged $\geq 18$ years, with T2DM for $\geq 12$ months prior to start of Ramadan fasting; $\mathrm{HbA} 1 \mathrm{c} \leq 8.5 \%$ (measured within 6 weeks prior to study entry); and treated with vildagliptin or SU either as dual therapy with metformin or as monotherapy for at least 4 weeks but not more than 3 years prior to commencement of fasting.

The study consisted of a 16-week observational period that included a fasting period of approximately 4 weeks. During this observational period, data from each patient were collected from at least two visits: at baseline (up to 6 weeks prior to the start of fasting, pre-Ramadan), and at the end of study (within 6 weeks after the end of fasting, post-Ramadan). In addition, data were collected if the patient made an interim visit during the fasting period.

\section{Study Assessments}

Primary assessment included the proportion of patients experiencing $\geq 1$ hypoglycemic event (HE), categorized as grade 1 (mild), defined as any reported symptoms by the patient and/or any blood glucose measurement $<3.9 \mathrm{mmol} / \mathrm{L}$, or grade 2 (severe), defined as the need for third-party assistance. Secondary assessments included change in HbA1c and body weight and treatment adherence from screening visit (baseline) to the end of the observational period. Safety assessments included monitoring and treatment of emergent adverse events (AEs) and serious adverse events (SAEs) as described by the physician. HEs based on blood glucose measurements and symptoms were recorded by patients in a diary. On failure to do so, HEs were recorded by the physician at the end of observational period, as recalled by the patient. Treatment adherence was based on the total number of fasting days and number of doses missed, if any, in those days. Any drug exposure during fasting was also recorded by patients in a diary during this period.

\section{Statistical Analysis}

The primary analysis set comprised all patients who received one dose of the study drug at the beginning of Ramadan and had at least one efficacy assessment after the start of fasting. The proportion of patients experiencing $\geq 1 \mathrm{HE}$ was analyzed using a two-sided Fisher's exact test. HbA1c assessment was also performed on the primary analysis set and was analyzed using a two sample $t$ test. Other assessments, such as change in body weight and reporting of AEs and SAEs were performed on the safety population, which consisted of all patients who received at least one dose of the study drug at the beginning of Ramadan and had at least one safety assessment. Change in body weight was analyzed using a two sample $t$ test. Missing data were computed using a last observation carried forward approach that carried the last post baseline observation forward to the end of the study. The $\mathrm{p}$ value reported in the study was descriptive in nature. Data analysis was performed by DATAMAP GmbH, Freiburg, Germany using SAS ${ }^{\circledR}$ Release 9.3 (SAS Institute Inc., Cary, NC, USA).

\section{Ethical Considerations}

Written informed consent to the collection and use of data was obtained from all participants, and the study was performed in accordance with the guidelines for good pharmacoepidemiological practices, national requirements and 
regulations, in line with the ethical principles laid down in the Declaration of Helsinki.

\section{RESULTS}

\section{Patient Disposition and Baseline Characteristics}

Of the 254 patients enrolled, 250 [121 (97.6\%) treated with vildagliptin, 125 (99.2\%) with SU and four (100\%) with other treatment] completed the study. Of these, 246 patients [121 (97.6\%) treated with vildagliptin and 125 $(99.2 \%)$ with SU] were included in the safety analysis set. Remaining four patients were lost to follow-up in the study; three (2.4\%) and one $(0.8 \%)$ in the vildagliptin and SU group, respectively.

The demographic and baseline characteristics of the patients by treatment group are presented in Table 1. Overall, approximately $56 \%$ of patients were men, mean patient age was 49 years, mean body weight was $89.9 \mathrm{~kg}$, mean body mass index (BMI) was $30.8 \mathrm{~kg} / \mathrm{m}^{2}$, and mean $\mathrm{HbA1c}$ at baseline was $7.5 \%$. A slight between-group variation was observed in the mean duration of T2DM (2.5 and 3.6 years in the vildagliptin and SU groups, respectively).

The median daily dose of antidiabetic drugs during the study is provided in Table 2 . The majority of the patients were receiving dual therapy with metformin; $90.9 \%(n=110 / 121)$ in the vildagliptin group and $68.8 \%(n=86 /$ $125)$ in the SU group. The mean (SD) duration of exposure to the study drug during Ramadan was 30.0 (0.0) and 29.8 (1.7) days in the vildagliptin and SU groups, respectively. Patients fasted for a mean (SD) of 29.7 (1.3) days in the vildagliptin group as compared to 29.3 (2.7) days in the SU group.

\section{Hypoglycemia}

During Ramadan, a significantly lower proportion of patients experienced $\geq 1$ HE in the vildagliptin group $(n=2 / 121,1.7 \%)$ than in the SU group $[n=24 / 125,19.2 \%(p<0.001)$; Table 3]. No patient in either group experienced a severe (grade 2) HE during Ramadan. None of the HEs led to discontinuation from the study.

\section{HbA1c and Body Weight Changes}

At the study endpoint, an $\mathrm{HbA1c}$ reduction of $0.1 \%$ was reported in the vildagliptin group (baseline $7.7 \%$ ) as compared to a $0.3 \%$ increase in the SU group (baseline 7.7\%), with a between-treatment difference of $-0.4 \%$ $(p<0.001)$ in favor of vildagliptin group (Fig. 1). Furthermore, vildagliptin group showed a reduction of $0.8 \mathrm{~kg}$ in body weight from pre- to post-Ramadan as compared to a reduction of $0.1 \mathrm{~kg}$ in the SU group, with a between-treatment difference of $-0.7 \mathrm{~kg}(p=0.011)$.

\section{Treatment Adherence}

Median daily dose of vildagliptin and SU throughout the study period remained the same (Table 2). Mean (SD) number of missed doses was $0.0 \quad(0.2)$ and $0.3 \quad(1.2)$ in the vildagliptin and SU groups, respectively. There was no medication change from pre-Ramadan to post-Ramadan in the vildagliptin group. A few patients required medication changes at pre-Ramadan [four (3.2\%)] and post-Ramadan [one (0.85\%)] in the SU group. 
Table 1 Patient demographic and baseline characteristics (safety set)

\begin{tabular}{lccc}
\hline Variable & Vildagliptin $\boldsymbol{n}=\mathbf{1 2 1}$ & Sulfonylurea $\boldsymbol{n}=\mathbf{1 2 5}$ & Total $\boldsymbol{N}=\mathbf{2 4 6}$ \\
\hline Age (years) & $48.3(7.5)$ & $49.7(8.9)$ & $49.0(8.2)$ \\
Age group, $n(\%)$ & & $239(97.2)$ \\
$<65$ years & $121(100)$ & $118(94.4)$ & $137(55.7)$ \\
Men $n(\%)$ & $73(60.3)$ & $64(51.2)$ & $89.9(14.4)$ \\
Weight $(\mathrm{kg})$ & $89.3(13.5)$ & $90.5(15.4)$ & $30.8(5.1)$ \\
BMI $\left(\mathrm{kg} / \mathrm{m}^{2}\right)$ & $30.5(4.9)$ & $31.0(5.2)$ & $3.0(2.4)$ \\
Duration of T2DM (years) & $2.5(1.6)$ & $3.6(2.9)$ & $7.5(0.8)$ \\
HbAlc $(\%)$ & $7.5(0.8)$ & $7.5(0.8)$ & $244(99.2)$ \\
Pre-Ramadan fasting advice, $n(\%)$ & $119(98.3)$ & $125(100.0)$ & $2(0.8)$ \\
Yes & $2(1.7)$ & $0(0)$ & $4(1.6)$ \\
No & $0(0)$ & $4(3.2)$ & $242(98.4)$ \\
Diabetes medication changed for fasting during Ramadan, $n(\%)$ & $121(96.8)$ & \\
Yes & $121(100)$ & & \\
No &
\end{tabular}

Values are mean $( \pm S D)$, unless indicated otherwise

$B M I$ body mass index, $S D$ standard deviation, T2DM type 2 diabetes mellitus, $n$ number of patients

Table 2 Daily dose of diabetes medication at the start and end of Ramadan fasting (safety set)

\begin{tabular}{|c|c|c|c|c|c|c|c|c|}
\hline & \multicolumn{4}{|c|}{ Vildagliptin $n=121$} & \multicolumn{4}{|c|}{ Sulfonylurea $n=125$} \\
\hline & \multicolumn{2}{|c|}{ Pre-Ramadan } & \multicolumn{2}{|c|}{ Post-Ramadan } & \multicolumn{2}{|c|}{ Pre-Ramadan } & \multicolumn{2}{|c|}{ Post-Ramadan } \\
\hline & $\overline{n^{\mathrm{a}}}$ & Median dose (mg) & $\overline{n^{\mathrm{a}}}$ & Median dose (mg) & $\overline{n^{\mathrm{a}}}$ & $\overline{\text { Median dose }(\mathrm{mg})}$ & $\overline{n^{\mathrm{a}}}$ & Median dose (mg) \\
\hline Metformin & 110 & 1700.0 & 110 & 1700.0 & 86 & 1000.0 & 86 & 1000.0 \\
\hline Vildagliptin & 121 & 100.0 & 121 & 100.0 & & & & \\
\hline Glibenclamide & & & & & 10 & 5.0 & 10 & 5.0 \\
\hline Gliclazide & & & & & 45 & 60.0 & 45 & 60.0 \\
\hline Glimepiride & & & & & 69 & 3.0 & 69 & 3.0 \\
\hline Glipizide & & & & & 1 & 10.0 & 1 & 10.0 \\
\hline
\end{tabular}

$n$ number of patients

a Patients with dosage information provided for the medication of interest

\section{Safety}

Overall incidence of AEs was higher in the SU group as compared to the vildagliptin group
$(23.2 \%$ vs. $5.8 \%$, respectively; Table 4$)$. The primary reason being the high incidence of hypoglycemia in the SU group $(n=24,19.2 \%)$. Other AEs reported were diarrhea, pyrexia and 
Table 3 Proportion of patients with hypoglycemic events during Ramadan fasting (primary analysis set)

\begin{tabular}{lllll}
\hline$n(\%)$ & $\begin{array}{l}\text { Vildagliptin } \\
\boldsymbol{n}=\mathbf{1 2 1}\end{array}$ & $\begin{array}{l}\text { Sulfonylurea } \\
\boldsymbol{n}=\mathbf{1 2 5}\end{array}$ & $\begin{array}{l}\text { Odds ratio (95\% } \\
\text { CI) }\end{array}$ & $\boldsymbol{p}$ value \\
\hline $\begin{array}{l}\text { Patients with grade 1 hypoglycemic } \\
\text { event }\end{array}$ & $2(1.7)$ & $24(19.2)^{\mathrm{a}}$ & $0.07(0.02,0.31)$ & $<0.001^{*}$ \\
$\begin{array}{l}\text { Patients with grade 2 hypoglycemic } \\
\text { event }\end{array}$ & 0 & 0 & - & - \\
\hline
\end{tabular}

$C I$ confidence interval, $n$ number of patients

${ }^{*}$ Fishers exact test

a glimepiride, 12/69 (17.4\%); gliclazide, 9/45 (20.0\%); glibenclamide, 3/10 (30.0\%); and glipizide, 0/1 (0.0\%)

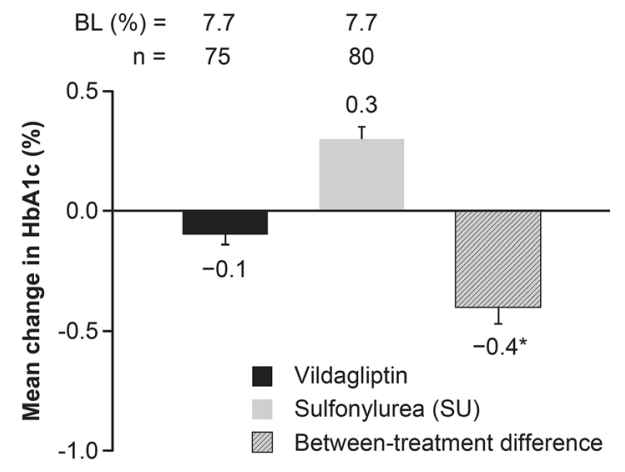

Fig. 1 Mean $( \pm S E)$ change in HbAlc pre- to postRamadan in vildagliptin and sulfonylurea groups (primary analysis set). ${ }^{*} p<0.001$. $B L$ baseline, $n$ number of patients, $S E$ standard error

headache. No SAEs or deaths were reported in either group. No patient in either group discontinued treatment due to an AE.

\section{DISCUSSION}

This prospective, observational study aimed to compare the incidences of hypoglycemia between vildagliptin and SU treatment in Muslim patients with T2DM who fasted during Ramadan in Egypt, represents a part of the already published and pooled data that included patients from Middle East and South Asia [19].
A significantly lower proportion of patients experienced hypoglycemia with vildagliptin as compared to SU $(1.7 \%$ vs. $19.2 \%, p<0.001)$. These findings are similar to those reported in the larger pooled analysis of the VIRTUE study [5.4\% vs. $19.8 \%$ with vildagliptin $(n=669)$ and SU $(n=621)$, respectively] [19]. In the randomized, controlled trial STudy Evaluating vildAgliptin compareD to gliclazide in patients with type 2 diabetes FASTing during Ramadan (STEADFAST; ClinicalTrials.gov identifier, NCT01758380), the risk of hypoglycemia was lower with vildagliptin than gliclazide; however, the difference was not statistically significant [20]. The authors attributed the small difference to the controlled study conditions, wherein patients received special attention in terms of good advice, treatment, and glycemic control, and therefore did not emulate a real-life setting, as observed in the VIRTUE study [19]. In addition, another randomized study reported no statistically significant difference $(p=0.334)$ in HEs between vildagliptin-treated patients and SU-treated patients during Ramadan which could be due to the timely follow-up with patients or change in their dietary habits during the fasting month [21]. 
Table 4 Overall summary of adverse events (safety set)

\begin{tabular}{lcc}
\hline Event, $\boldsymbol{n}(\%)$ & Vildagliptin $\boldsymbol{n = 1 2 1}$ & Sulfonylurea $\boldsymbol{n}=\mathbf{1 2 5}$ \\
\hline Any AE & $7(5.8)$ & $29(23.2)$ \\
Any SAE & $0(0.0)$ & $0(0.0)$ \\
Discontinuation due to AEs & $0(0.0)$ & $0(0.0)$ \\
Deaths & $0(0.0)$ & $0(0.0)$ \\
Most common AEs by preferred term $(\geq 1.7 \%$ in any group) & \\
Diarrhea & $3(2.5)$ & $0(0.0)$ \\
Hypoglycemia & $2(1.7)$ & $24(19.2)$ \\
Pyrexia & $2(1.7)$ & $4(3.2)$ \\
\hline
\end{tabular}

$A E$ adverse event, $n$ number of patients, $S A E$ serious adverse event

The present results also concur with the findings from larger pooled analysis of the VIRTUE study as well as several observational studies conducted in patients fasting during Ramadan across various parts of the world and following different types of diet and lifestyle habits [14-20, 22]. In these studies, vildagliptin consistently showed a lower incidence of hypoglycemia compared with SUs (including glimepiride, gliclazide, glibenclamide, and glipizide). Vildagliptin also showed better treatment adherence and improved glycemic control as compared to SUs when administered as dual therapy with metformin [14-20, 22].

Vildagliptin improves glycemic control by causing glucose-dependent insulin secretion through inhibition of the DPP-4 enzyme, thereby increasing the sensitivity and responsiveness of $\beta$ - and $\alpha$-cells to glucose $[12,23]$. Vildagliptin exhibiting a low risk of hypoglycemia is likely due to the fact that both meal-dependent insulin secretion and suppression of glucagon secretion are glucose-dependent [11, 24]. The favorable outcome observed during fasting is due to the suppression of inappropriate glucagon secretion during hyperglycemia as well as the enhancement of glucose-dependent insulinotropic polypeptide (GIP)-mediated effect on glucagon, which results in protection against hypoglycemia. The levels of both glucagon-like peptide-1 (GLP-1) and GIP remain high during the inter-meal and overnight periods, when hypoglycemia is more likely to occur [12].

Similar findings have been reported with another DPP-4 inhibitor, sitagliptin, in randomized pragmatic studies [25, 26]. Results from studies with vildagliptin and sitagliptin suggest that DPP-4 inhibitors are safe and effective treatment options for T2DM patients fasting during Ramadan [20, 25].

Vildagliptin was associated with a moderate, but significant reduction in $\mathrm{HbA1c}$ from baseline as compared to $\mathrm{SU}$, with a between-group difference of $-0.4 \%(p<0.001)$. This finding is consistent with other vildagliptin studies conducted in fasting patients with T2DM $[14,19]$. Results from this study showed that vildagliptin conferred small reductions in body weight in patients with T2DM who fasted during Ramadan. This finding is in agreement with previous studies $[14,18]$ and could be attributed to the low 
incidence of hypoglycemia which might not have led to defensive eating.

Overall, both groups showed complete treatment adherence in this study which is in agreement with the high treatment adherence observed in the VIRTUE study's pooled results, with a low and similar number of missed doses (0.7 and 0.8 in the vildagliptin and SU groups, respectively) [19].

This study had several limitations. First, being an observational study, there are inherent limitations and bias that accompany results of observational study works. Second, blood glucose measurements were not required to confirm the hypoglycemic episodes as assessed by the primary endpoint ( $\geq 1 \mathrm{HE}$ ). Thus, the lack of a confirmatory glucose measurement may have overestimated the incidence of hypoglycemia.

\section{CONCLUSIONS}

Treatment with vildagliptin was associated with lower incidence of hypoglycemia when compared with SU in patients who fast during month of Ramadan. Furthermore, vildagliptin showed good glycemic and weight control and was well tolerated in patients with T2DM fasting during Ramadan in a real-life setting in Egypt.

\section{ACKNOWLEDGMENTS}

The pooled study was sponsored by Novartis Pharma AG, Basel, Switzerland. The present analysis was carried out by authors with assistance from Novartis Pharma, Cairo, Egypt with regards to conduct of the study, collecting, compiling and conceptualizing data (data were analyzed by the CRO, DATAMAP GmbH, in Freiburg, Germany). All named authors meet the International Committee of Medical Journal Editors criteria for authorship for this manuscript, take responsibility for the integrity of the work as a whole, and have given final approval to the version to be published. The article processing charges were funded by Novartis Pharma, Cairo, Egypt. The authors thank Nashwa Nashaat (former employee of Novartis Pharma, Egypt) for her contribution in reviewing the study protocol and manuscript outline. Medical writing assistance in the preparation of this manuscript was provided by Shilpa Kakkar and Anuja Shah (both form Novartis Healthcare Private Ltd., Hyderabad, India) and was supported by Novartis Pharma, Cairo, Egypt. The authors would like to thank Abhilasha Verma (Novartis Healthcare Private Limited, Hyderabad, India) for editorial assistance, collation and incorporation of comments from all the authors.

Disclosures. M. Khattab has no conflict of interest. K. Mahmoud has received honoraria for lectures and advisory boards from Eli Lilly, Novartis Pharmaceuticals, AstraZeneca, GSK, Novo Nordisk, Servier, Boehringer Ingelheim, Pfizer, Merck Serono, Merck Sharp \& Dohme Limited, Sanofi-Aventis and Abbott. I. Shaltout has no conflict of interest. M. Khattab, K. Mahmoud, and I. Shaltout. have received investigator fees related to the conduct of the VIRTUE study from Novartis and its affiliates.

Compliance with Ethics Guidelines. All procedures followed were in accordance with the ethical standards of the responsible committee on human experimentation (institutional and national) and with the Helsinki Declaration of 1975, as revised in 2000 and 2008. Informed consent was obtained from all patients included in the study. 
Open Access. This article is distributed under the terms of the Creative Commons Attribution-NonCommercial 4.0 International License (http://creativecommons.org/licenses/ by-nc/4.0/), which permits any noncommercial use, distribution, and reproduction in any medium, provided you give appropriate credit to the original author(s) and the source, provide a link to the Creative Commons license, and indicate if changes were made.

\section{REFERENCES}

1. International Diabetes Federation Middle East and North Africa; 2015. http://www.idf.org/membership/ mena/egypt. Accessed 4 Apr 2016.

2. International Diabetes Federation. IDF Diabetes Atlas. 7th ed. Brussels, Belgium: International Diabetes Federation;2015. http://www.idf.org/ diabetesatlas. Accessed 4 Apr 2016.

3. The Future of the Global Muslim Population. Pew research center Religion \& Public Life;2011. http://www.pewforum.org/interactives/muslimpopulation-graphic/. Accessed 4 Apr 2016.

4. Al-Arouj M, Assaad-Khalil S, Buse J, et al. Recommendations for management of diabetes during Ramadan: update 2010. Diabetes Care. 2010;33:1895-902.

5. Bashir MI, Pathan MF, Raza SA, et al. Role of oral hypoglycemic agents in the management of type 2 diabetes mellitus during Ramadan. Indian J Endocrinol Metab. 2012;16:503-7.

6. Jaleel MA, Raza SA, Fathima FN, Jaleel BN. Ramadan and diabetes: As-Saum (The fasting). Indian J Endocrinol Metab. 2011;15:268-73.

7. Hui E, Bravis V, Hassanein M, et al. Management of people with diabetes wanting to fast during Ramadan. Br Med J. 2010;340:c3053.

8. Salti I, Benard E, Detournay B, et al. A population-based study of diabetes and its characteristics during the fasting month of Ramadan in 13 countries: results of the epidemiology of diabetes and Ramadan 1422/2001 (EPIDIAR) study. Diabetes Care. 2004;27:2306-11.

9. Ahmad J, Pathan MF, Jaleel MA, et al. Diabetic emergencies including hypoglycemia during
Ramadan. Indian J Endocrinol Metab. 2012;16:512-5.

10. Almaatouq MA. Pharmacological approaches to the management of type 2 diabetes in fasting adults during Ramadan. Diabetes Metab Syndr Obes. 2012;5:109-19.

11. Ahrén BB, Schweizer A, Dejager S, et al. Vildagliptin enhances islet responsiveness to both hyper- and hypoglycemia in patients with type 2 diabetes. J Clin Endocrinol Metab. 2009;94:1236-43.

12. Ahrén B, Foley JE, Bosi E. Clinical evidence and mechanistic basis for vildagliptin's action when added to metformin. Diabetes Obes Metab. 2011;13:193-203.

13. Dejager S, Schweizer A. Minimizing the risk of hypoglycemia with vildagliptin: clinical experience, mechanistic basis, and importance in type 2 diabetes management. Diabetes Ther. 2011;2:51-66.

14. Hassanein M, Hanif W, Malik W, et al. Comparison of the dipeptidyl peptidase- 4 inhibitor vildagliptin and the sulfonylurea gliclazide in combination with metformin, in Muslim patients with type 2 diabetes mellitus fasting during Ramadan: results of the VECTOR study. Curr Med Res Opin. 2011;27:1367-74.

15. Hanif W, Malik W, Hassanein M, et al. Treatment adherence with vildagliptin compared to sulfonylurea as add-on to metformin in Muslim patients with type 2 diabetes mellitus fasting during Ramadan. Curr Med Res Opin. 2013;29:807-11.

16. Shete A, Shaikh A, Nayeem KJ, et al. Vildagliptin vs sulfonylurea in Indian Muslim diabetes patients fasting during Ramadan. World J Diabetes. 2013;4:358-64.

17. Devendra D, Gohel B, Bravis V, et al. Vildagliptin therapy and hypoglycemia in Muslim type 2 diabetes patients during Ramadan. Int $\mathrm{J}$ Clin Pract. 2009;63:1446-50.

18. Halimi S, Levy $M$, Huet D, Quéré S, Dejager S. Experience with vildagliptin in type 2 diabetic patients fasting during Ramadan in France: insights from the VERDI study. Diabetes Ther. 2013;4:385-98.

19. Al-Arouj M, Hassoun AA, Medlej R, et al. The effect of vildagliptin relative to sulfonylureas in Muslim patients with type 2 diabetes fasting during Ramadan: the VIRTUE study. Int J Clin Pract. 2013;67:957-63.

20. Hassanein M, Abdallah $\mathrm{K}$, Schweizer A. A double-blind, randomized trial, including frequent patient-physician contacts and Ramadan-focused advice, assessing vildagliptin and gliclazide in 
patients with type 2 diabetes fasting during Ramadan: the STEADFAST study. Vasc Health Risk Manag. 2014;10:319-26.

21. Malha LP, Taan G, Zantout MS, Azar ST. Glycemic effects of vildagliptin in patients with type 2 diabetes before, during and after the period of fasting in Ramadan. Ther Adv Endocrinol Metab. 2014;5:3-9.

22. Schweizer A, Halimi S, Dejager S. Experience with DPP-4 inhibitors in the management of patients with type 2 diabetes fasting during Ramadan. Vasc Health Risk Manag. 2014;10:15-24.

23. Mari A, Sallas WM, He YL, et al. Vildagliptin, a dipeptidyl peptidase-IV inhibitor, improves model-assessed beta-cell function in patients with type 2 diabetes. J Clin Endocrinol Metab. 2005;90:4888-94.
24. Schweizer A, Foley JE, Kothny W, Ahrén B. Clinical evidence and mechanistic basis for vildagliptin's effect in combination with insulin. Vasc Health Risk Manag. 2013;9:57-64.

25. Al Sifri S, Basiounny A, Echtay A, et al. The incidence of hypoglycemia in Muslim patients with type 2 diabetes treated with sitagliptin or a sulfonylurea during Ramadan: a randomized trial. Int J Clin Pract. 2011;65:1132-40.

26. Aravind SR, Ismail SB, Balamurugan $\mathrm{R}$, et al. Hypoglycemia in patients with type 2 diabetes from India and Malaysia treated with sitagliptin or a sulfonylurea during Ramadan: a randomized, pragmatic study. Curr Med Res Opin. 2012;28:1289-96. 